\title{
Some inequalities involving multivalent functions
}

\author{
by Shigeyoshi Owa (Osaka), \\ Mamoru NunokaWa (Gunma) and \\ Hitoshi SAitoh (Gunma)
}

Abstract. The object of the present paper is to derive some inequalities involving multivalent functions in the unit disk. One of our results is an improvement and a generalization of a result due to R. M. Robinson [4].

1. Introduction. Let $\mathbb{A}(p)$ be the class of functions of the form

$$
f(z)=z^{p}+\sum_{n=p+1}^{\infty} a_{n} z^{n} \quad(p \in \mathbb{N}=\{1,2,3, \ldots\})
$$

which are analytic in the unit disk $\mathbb{U}=\{z:|z|<1\}$.

In 1947, Robinson [4] proved the following

Theorem A. Let $S(z)$ and $T(z)$ be analytic in $\mathbb{U}$, and let $\operatorname{Re}\left\{z S^{\prime}(z) / S(z)\right\}>0(z \in \mathbb{U})$. If $\left|T^{\prime}(z) / S^{\prime}(z)\right|<1(z \in \mathbb{U})$ and $T(0)=0$, then $|T(z) / S(z)|<1(z \in \mathbb{U})$.

In the present paper, we derive an improvement and generalization of Theorem $\mathrm{A}$ for functions belonging to $\mathbb{A}(p)$.

To establish our results, we have to recall the following lemmas.

Lemma 1 ([1], [2]). Let $w(z)$ be analytic in $\mathbb{U}$ with $w(0)=0$. If $|w(z)|$ attains its maximum value in the circle $|z|=r<1$ at a point $z_{0} \in \mathbb{U}$, then we can write

$$
z_{0} w^{\prime}\left(z_{0}\right)=k w\left(z_{0}\right)
$$

where $k$ is real and $k \geq 1$.

Lemma 2 ([3]). Let $p(z)$ be analytic in $\mathbb{U}$ with $p(0)=1$. If there exists a

1991 Mathematics Subject Classification: Primary 30C45.

Key words and phrases: analytic function, unit disk, Jack's lemma. 
point $z_{0} \in \mathbb{U}$ such that

$\operatorname{Re}(p(z))>0 \quad\left(|z|<\left|z_{0}\right|\right), \quad \operatorname{Re}\left(p\left(z_{0}\right)\right)=0, \quad$ and $\quad p\left(z_{0}\right) \neq 0$, then $p\left(z_{0}\right)=i a(a \neq 0)$ and

$$
\frac{z_{0} p^{\prime}\left(z_{0}\right)}{p\left(z_{0}\right)}=i \frac{k}{2}\left(a+\frac{1}{a}\right)
$$

where $k$ is real and $k \geq 1$.

2. Some counterparts of Theorem A. Our first result for functions in the class $\mathbb{A}(p)$ is contained in

Theorem 1. Let $S(z) \in \mathbb{A}(m), T(z) \in \mathbb{A}(n)$ with $p=n-m \geq 1$. Let $S(z)$ satisfy $\operatorname{Re}\left\{S(z) / z S^{\prime}(z)\right\}>\alpha(0 \leq \alpha<1 / m)$. If

$$
\left|\frac{T^{\prime}(z)}{S^{\prime}(z)}\right|<(1+p \alpha)|z|^{p-1} \quad(z \in \mathbb{U})
$$

then

$$
\left|\frac{T(z)}{S(z)}\right|<|z|^{p-1} \quad(z \in \mathbb{U}) .
$$

Proof. Since $T(z) / S(z)=z^{p}+\ldots \in \mathbb{A}(p)$, we define the function $w(z)$ by $T(z)=z^{p-1} w(z) S(z)$. Then $w(z)$ is analytic in $\mathbb{U}$ with $w(0)=0$. It follows from the definition of $w(z)$ that

$$
\frac{T^{\prime}(z)}{S^{\prime}(z)}=z^{p-1} w(z)\left\{1+\left(p-1+\frac{z w^{\prime}(z)}{w(z)}\right) \frac{S(z)}{z S^{\prime}(z)}\right\} .
$$

If we suppose that there exists a point $z_{0} \in \mathbb{U}$ such that

$$
\max _{|z| \leq\left|z_{0}\right|}|w(z)|=\left|w\left(z_{0}\right)\right|=1,
$$

then Lemma 1 gives $w\left(z_{0}\right)=e^{i \theta}$ and

$$
z_{0} w^{\prime}\left(z_{0}\right)=k w\left(z_{0}\right) \quad(k \geq 1) .
$$

Therefore,

$$
\begin{aligned}
\left|\frac{T^{\prime}\left(z_{0}\right)}{z_{0}^{p-1} S^{\prime}\left(z_{0}\right)}\right| & =\left|1+\left(p-1+\frac{z_{0} w^{\prime}\left(z_{0}\right)}{w\left(z_{0}\right)}\right) \frac{S\left(z_{0}\right)}{z_{0} S^{\prime}\left(z_{0}\right)}\right| \\
& \geq 1+(p-1+k) \operatorname{Re}\left(\frac{S\left(z_{0}\right)}{z_{0} S^{\prime}\left(z_{0}\right)}\right)>1+p \alpha .
\end{aligned}
$$

This contradicts our condition (2.1), so that $|w(z)|<1$ for all $z \in \mathbb{U}$. This completes the proof of Theorem 1.

Remark. If we take $p=1$ and $\alpha=0$ in Theorem 1 , then we recover Theorem A due to Robinson [4].

Next, applying Lemma 2, we prove 
Theorem 2. Let $S(z) \in \mathbb{A}(m), T(z) \in \mathbb{A}(n)$ with $p=n-m \geq 1$. Let $S(z)$ satisfy $\operatorname{Re}\left\{S(z) / z S^{\prime}(z)\right\}>\alpha(0 \leq \alpha<1 / m)$ and $-\alpha / p \leq \operatorname{Im}\left\{S(z) /\left(z S^{\prime}(z)\right)\right\}$ $\leq \alpha / p(0 \leq \alpha<1 / m)$. If

$$
\operatorname{Re}\left(\frac{T^{\prime}(z)}{z^{p} S^{\prime}(z)}\right)>0 \quad(z \in \mathbb{U})
$$

then

$$
\operatorname{Re}\left(\frac{T(z)}{z^{p} S(z)}\right)>0 \quad(z \in \mathbb{U}) .
$$

Pro of. Defining the function $q(z)$ by $T(z)=z^{p} q(z) S(z)$, we see that $q(z)$ is analytic in $\mathbb{U}$ with $q(0)=1$. Note that

$$
\frac{T^{\prime}(z)}{S^{\prime}(z)}=z^{p} q(z)\left\{1+\left(p+\frac{z q^{\prime}(z)}{q(z)}\right) \frac{S(z)}{z S^{\prime}(z)}\right\} .
$$

Suppose that there exists a point $z_{0} \in \mathbb{U}$ such that

$$
\operatorname{Re}(q(z))>0 \quad\left(|z|<\left|z_{0}\right|\right), \quad \operatorname{Re}\left(q\left(z_{0}\right)\right)=0, \quad \text { and } \quad q\left(z_{0}\right) \neq 0 .
$$

Then, applying Lemma 2 , we have $q\left(z_{0}\right)=i a(a \neq 0)$ and

$$
\frac{z_{0} q^{\prime}\left(z_{0}\right)}{q\left(z_{0}\right)}=i \frac{k}{2}\left(a+\frac{1}{a}\right) \quad(k \geq 1) .
$$

Therefore, writing $S\left(z_{0}\right) /\left(z_{0} S^{\prime}\left(z_{0}\right)\right)=\alpha_{0}+i \beta_{0}$, we obtain

$$
\begin{aligned}
\operatorname{Re}\left(\frac{T^{\prime}\left(z_{0}\right)}{z_{0}^{p} S^{\prime}\left(z_{0}\right)}\right) & =-a p \beta_{0}-\frac{a k \alpha_{0}}{2}\left(a+\frac{1}{a}\right) \\
& =-a p \beta_{0}-\frac{k \alpha_{0}}{2}\left(1+a^{2}\right) \\
& \leq-a p \beta_{0}-\frac{\alpha_{0}}{2}\left(1+a^{2}\right) \leq-a p \beta_{0}-\frac{\alpha}{2}\left(1+a^{2}\right) .
\end{aligned}
$$

Since $-\alpha / p \leq \beta_{0} \leq \alpha / p$, if $a>0$, then

$$
\begin{aligned}
-a p \beta_{0}-\frac{\alpha}{2}\left(1+a^{2}\right) & \leq a \alpha-\frac{\alpha}{2}\left(1+a^{2}\right) \\
& =-\frac{\alpha}{2}(1-a)^{2} \leq 0,
\end{aligned}
$$

and if $a<0$, then

$$
\begin{aligned}
-a p \beta_{0}-\frac{\alpha}{2}\left(1+a^{2}\right) & \leq-a \alpha-\frac{\alpha}{2}\left(1+a^{2}\right) \\
& =-\frac{\alpha}{2}(1+a)^{2} \leq 0 .
\end{aligned}
$$

This contradicts our condition (2.5). Consequently, $\operatorname{Re}(q(z))>0$ for all $z \in \mathbb{U}$, so that $\operatorname{Re}\left\{T(z) /\left(z^{p} S(z)\right)\right\}>0\left(z_{0} \in \mathbb{U}\right)$.

Further, using the same technique as in the proof of Theorem 2, we obtain 
Theorem 3. Let $S(z) \in \mathbb{A}(m), T(z) \in \mathbb{A}(n)$ with $p=m-n$ $\geq 0$. Let $S(z)$ satisfy $\operatorname{Re}\left\{S(z) /\left(z S^{\prime}(z)\right)\right\}>\alpha(0 \leq \alpha<1 / m)$ and $-\alpha / p \leq$ $\operatorname{Im}\left\{S(z) /\left(z S^{\prime}(z)\right)\right\} \leq \alpha / p(0 \leq \alpha<1 / m)$. If

$$
\operatorname{Re}\left(\frac{z^{p} T^{\prime}(z)}{S^{\prime}(z)}\right)>0 \quad(z \in \mathbb{U})
$$

then

$$
\operatorname{Re}\left(\frac{z^{p} T(z)}{S(z)}\right)>0 \quad(z \in \mathbb{U})
$$

Acknowledgements. This research was supported in part by the Japanese Ministry of Education, Science and Culture under Grant-in-Aid for General Scientific Research.

\section{References}

[1] I. S. Jack, Functions starlike and convex of order $\alpha$, J. London Math. Soc. 3 (1971), 469-474.

[2] S. S. Miller and P. T. Mocanu, Second order differential inequalities in the complex plane, J. Math. Anal. Appl. 65 (1978), 289-305.

[3] M. Nunokawa, On properties of non-Carathéodory functions, Proc. Japan Acad. 68 (1992), 152-153.

[4] R. M. Robinson, Univalent majorants, Trans. Amer. Math. Soc. 61 (1947), 1-35.

S. Owa

DEPARTMENT OF MATHEMATICS

KINKI UNIVERSITY

HIGASHI-OSAKA, OSAKA 577

JAPAN

M. Nunokawa

DEPARTMENT OF MATHEMATICS

UNIVERSITY OF GUNMA

ARAMAKI, MAEBASHI, GUNMA 371

JAPAN
H. Saitoh DEPARTMENT OF MATHEMATICS GUNMA COLLEGE OF TECHNOLOGY TORIBA, MAEBASHI, GUNMA 371 